\title{
Durées de vie, processus endoénergétiques et potentiels de l'exciplexe Kr*Ar
}

\author{
F. Bourgeois, R. Reininger, P. Gürtler*, J.L. Subtil**, C. Jonin** et P. Laporte** \\ Synchrotron Radiation Center, University of Wisconsin, Madison, 3731 Schneider Dr., \\ Stoughton, WI 53589, U.S.A. \\ * Hamburger Synchrotronstrahlungslabor (HASYLAB) at DESY, Notkestr. 85, \\ 2000 Hamburg 52, Allemagne \\ ** Laboratoire de Traitement du Signal et Instrumentation (LTSI), CNRS-UMR 5516, \\ Université Jean Monnet, 23 rue du Dr. P. Michelon, 42023 Saint-Etienne cedex 2, \\ France
}

\begin{abstract}
Résumé : La cinétique et la spectroscopie de l'exciplexe $\mathrm{Kr}^{*} \mathrm{Ar}$ ont été étudiées. Les résultats experimentaux démontrent des effets de température marqués avec mise en évidence de processus collisionnels endoénergétiques. La profondeur des puits de potentiel de l'exciplexe est estimée à $1150 \mathrm{~cm}^{-1}$ et les durées de vie des états $0^{+}$et $1,0^{-}$sont déduites avec pour valeurs respectives 3,2 et 210 ns.
\end{abstract}

La cinétique de déclin des mélanges de gaz rares reste mal connue du fait notamment du rôle considérable des collisions entre atomes identiques qui masquent bien souvent l'effet des interactions spécifiquement hétéronucléaires. De plus et de ce fait, des grandeurs de base comme les durées de vie radiatives des exciplexes n'ont pas encore été déterminées précisément.

A l'aide de techniques décrites précédemment [1, 2] (rayonnement synchrotron, dopage dans la gamme du p.p.m.) et d'outils d'analyse adaptes, nous sommes parvenus aux résultats et conclusions suivants pour l'exciplexe $\mathrm{Kr}^{*} \mathrm{Ar}$. A température ambiante, l'émission dite "lente" de l'exciplexe $\left(1,0^{-}\right)$qui fait suite à l'emission dite "rapide" $\left(0^{+}\right)$n'est pas l'unique canal de déclin. En effet, moyennant des conditions expérimentales appropriées, un déclin synchrone est observé au niveau de l'émission ${ }^{3} \mathrm{P}_{1}(\mathrm{Kr}) \rightarrow{ }^{1} \mathrm{~S}_{0}$.

Ceci explique que ces états restent couplés collisionnellement et que des collisions fortement endoénergétiques peuvent ramener de l'exciplexe $1,0^{-}$au niveau ${ }^{3} \mathrm{P}_{1}$ (Fig.1). Les effets de température sur la bande d'émission de l'exciplexe ont permis d'évaluer les constantes spectroscopiques associées aux puits de potentiel des états $1,0^{-}$et $0^{+}$. La profondeur de ceux-ci est évalué à $1150 \mathrm{~cm}^{-1}$. Ces effets sont également très marqués en œe qui concerne la dynamique de déclin. En effet, on observe à température ambiante (Fig.2a) deux temps de déclin strictement identiques pour les émissions bien distinctes de l'exciplexe et des atomes de krypton, qui s'établissent après l'émission dite rapide. De tels effets montrent indubitablement l'efficacité de ces processus endoénergétiques malgré les écarts énergétiques mis en jeu ( de l'ordre de $2000 \mathrm{~cm}^{-1}$ entre les énergies du niveau ${ }^{3} \mathrm{P}_{1}$ et du fond du puits des états $1,0^{-}$). On constate à basse température (Fig.2b) la disparition totale de la composante lente associée au déclin atomique, traduisant l'inhibition progressive des collisions endoénergétiques.

De tels résultats impliquent une modélisation d'ensemble très complexe nécessitant un grand volume de données en cours d'analyse. Des résultats préliminaires obtenus à partir de modèles simplifiés conduisent aux durées de vie radiatives des exciplexes $0^{+}$et $1,0^{-}$de 3,2 et $210 \mathrm{~ns}$, respectivement. Une modélisation plus complète est cependant de nature à modifier légèrement la valeur obtenue pour la composante rapide. 
Remerciements : Ce travail a reçu le support OTAN reff. CRG 920574.

\section{Références :}

[1] Laporte P., Subtil J.L., Reininger R., and Gürtler P.

Chem. Phys. 177, (1993) 257-269

[2] Laporte P., Subtil J.L., Reininger R., and Gürtler P.

Phys. Rev. Lett. 74, (1995) 1954-1957

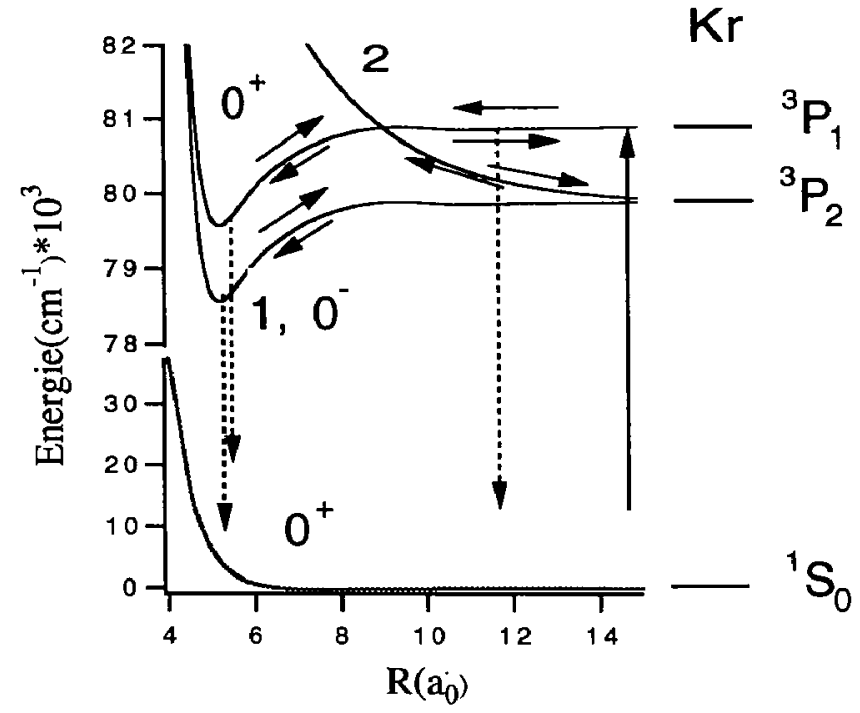

Figure 1 : Schéma cinétique de formation de l'exciplexe $\mathrm{Kr}^{*} \mathrm{Ar}$
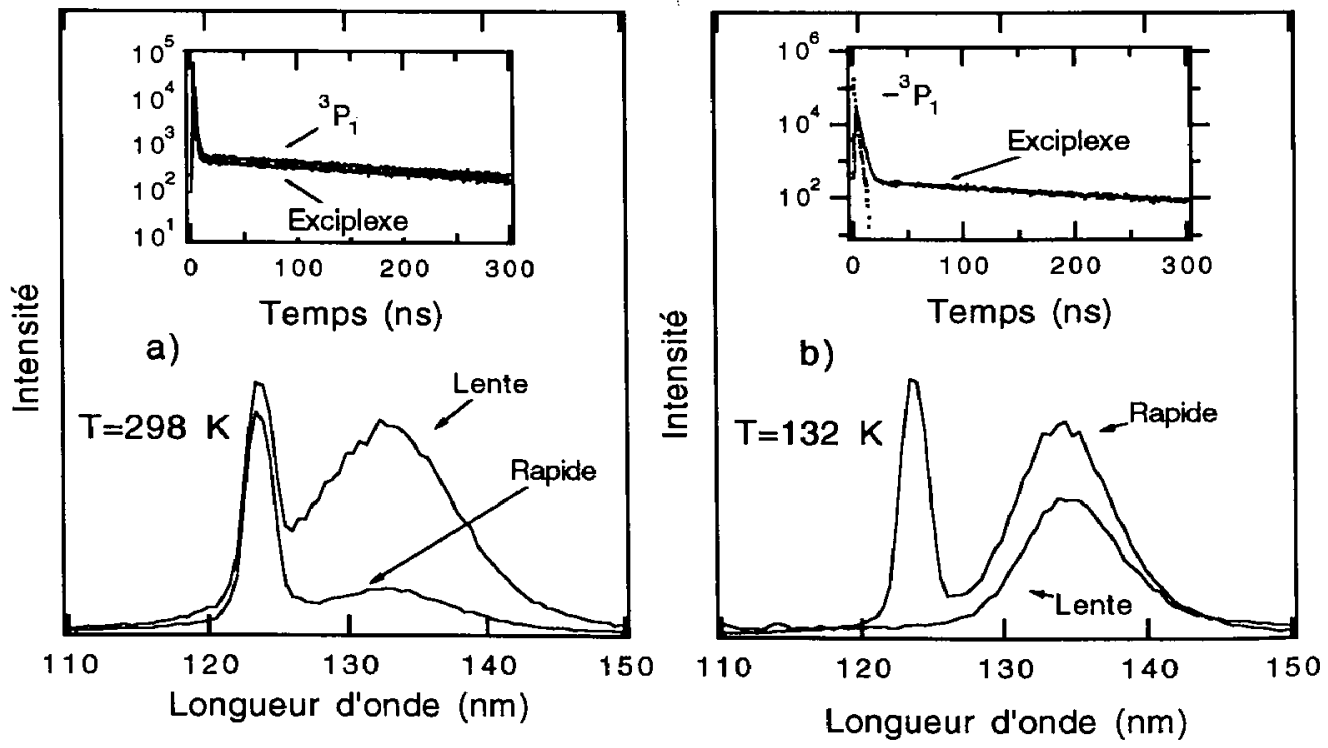

Figure 2 : Spectroscopie et déclin radiatif de l'exciplexe $\mathrm{Kr}^{*} \mathrm{Ar}$ 